\title{
Manejo de pacientes con síndrome mielodisplásico en época COVID-19
}

\section{Elia Apodaca1*, Carlos R. Hernández-Pérez² y Ma. Guadalupe Rodríguez-González ${ }^{3}$}

${ }^{1}$ Departamento de Hematología, Instituto Nacional de Ciencias Médicas y Nutrición Salvador Zubirán; ²Departamento de Hematología, Hospital de Especialidades del CMN Siglo XXI, IMSS; ${ }^{3}$ Departamento de Hematología, Hospital de Especialidades CMN La Raza, IMSS. Ciudad de México, Méx., México

\section{Resumen}

La infección por coronavirus 2 del síndrome respiratorio agudo grave ha provocado un cambio en la forma de atender a los pacientes con enfermedades hematológicas en todo el mundo. Los pacientes con síndrome mielodisplásico (SMD) se han visto afectados por la ausencia de conocimiento del comportamiento de la enfermedad por coronavirus 2019 (COVID-19) en este tipo de padecimiento. Se han establecido lineamientos internacionales que han permitido continuar con la atención de dichos pacientes. El principal objetivo de esta revisión es definir las medidas preventivas y las estrategias de tratamiento que se deben de tomar al momento de evaluar a un paciente con SMD en la época COVID-19.

PALABRAS CLAVE: COVID-19. Síndrome mielodisplásico. Pacientes.

\section{Management of patients with Myelodysplastic Syndrome in the COVID-19 era}

\begin{abstract}
SARS-CoV-2 infection has caused a change in the way we care for patients with hematological diseases around the world. Patients with myelodysplastic syndrome (MDS) have been affected by the lack of knowledge of the behavior of COVID-19 in this type of condition. International guidelines have been established that have made it possible to continue caring for these patients. The main objective of this review is to define the preventive measures and treatment strategies that should be taken when evaluating a patient with myelodysplastic syndrome in the COVID-19 era.
\end{abstract}

KEY WORDS: COVID-19. Myelodysplastic syndrome (MDS). Patients.

\section{Introducción}

El brote de la enfermedad por coronavirus 2019 (COVID-19) ha planteado un desafío sin precedentes para los servicios médicos de todo el mundo. La COVID19 está causada por la infección por el coronavirus 2 del síndrome respiratorio agudo grave (SARS-CoV-2), un nuevo betacoronavirus del mismo subgénero del virus del síndrome respiratorio agudo severo (SARS). El 11 de marzo de 2020 la Organización Mundial de la Salud (OMS) declaró que el mundo se encontraba en pandemia, debido a que más del $50 \%$ de la comunidad mundial tenía posibilidades de infectarse con el nuevo coronavirus'. La enfermedad se ha expandido rápidamente, se ha informado un total de 9,843,073 casos confirmados de COVID-19 en el mundo, con 495,760 muertes, hasta el 28 de junio de $2020^{2}$.
Correspondencia:

*Elia Apodaca

E-mail: eliapandaoo@gmail.com
Fecha de recepción: 09-09-2020

Fecha de aceptación: 09-02-2021

DOI: 10.24875/GMM.M21000470
Gac Med Mex. 2021;157(Supl 3):S47-S51

Disponible en PubMed

www.gacetamedicademexico.com

0016-3813/@ 2021 Academia Nacional de Medicina de México, A.C. Publicado por Permanyer. Este es un artículo open access bajo la licencia CC BY-NC-ND (http://creativecommons.org/licenses/by-nc-nd/4.0/). 
En el mundo se están viendo diferentes niveles de actividad de COVID-19 con respecto a la tasa de infección, la población susceptible y la tasa de mortalidad. Los países con mayor número de casos son Ios siguientes: EE.UU., Brasil, Federación Rusa, India, Gran Bretaña, Perú, Chile, España, Italia, Irán, México y Pakistán ${ }^{2}$. Actualmente México ocupa el 11. o lugar mundial en contagios y el 5. lugar de las Américas, con 208,392 casos confirmados y 25,779 muertes $^{2}$.

Los síntomas son muy variables, desde asintomáticos hasta progresar a insuficiencia respiratoria aguda. En las diversas series de pacientes con COVID se describen los siguientes síntomas: fiebre $(>90 \%)$, tos (> 70\%), disnea (>50\%), mialgias o fatiga $(>40 \%)$, expectoración $(28 \%)$, cefalea $(8 \%)$, hemoptisis $(5 \%)$ y síntomas gastrointestinales $(3-10 \%)^{3-5}$. Un $29 \%$ progresan a síndrome respiratorio agudo, un $12 \%$ a daño cardiaco agudo, un $7 \%$ a lesión renal aguda y la mortalidad reportada es de un $4-11 \%$.

El cuidado de los pacientes con cáncer ha cambiado extremadamente con la llegada de esta pandemia. Datos de China muestran que los pacientes con cáncer con COVID-19 tienen 3.56 (intervalo de confianza del 95\%: 1.65-7.69) veces más riesgo de requerir ventilación mecánica invasiva o ingreso a terapia intensiva que la población en general ${ }^{6}$. También se ha reportado mayor tasa de mortalidad en pacientes con cáncer (28.6\%), sin embargo, en estos estudios no se incluyeron pacientes con padecimientos hematológicos ${ }^{7,8}$. La razón de pensar que el riesgo es semejante en pacientes hematológicos es por el empleo de quimioterapia, inmunosupresión, uso de esteroides y exposición frecuente a los centros hospitalarios 7,8 .

Se realizó una búsqueda sistematizada en PubMed y no existen estudios donde se describa la prevalencia, factores de riesgo y mortalidad de pacientes con síndrome mielodisplásico (SMD) infectados por SARS-CoV-2, pero independientemente de esto son pacientes que seguimos diagnosticando y en los que estamos tomando decisiones de tratamiento durante esta pandemia.

\section{Experiencia mundial}

No existen estudios donde se demuestre que en pacientes con SMD el riesgo de infección por SARSCoV-2 sea mayor que en personas sin SMD; la inmunodeficiencia en el SMD está principalmente relacionada con los linajes mieloides y monocíticos. Muchos pacientes con SMD tienen neutropenia 0 neutropenia funcional, lo cual incrementa el riesgo de infecciones bacterianas y fúngicas, en mayor medida que las infecciones virales ${ }^{3-6}$.

Dentro del grupo de pacientes con SMD los que tienen mayor riesgo de infecciones virales son los que fueron tratados con globulina antitimocito o trasplante de células progenitoras hematopoyéticas (TCPH) alogénico; desde el punto de vista fisiopatológico sería este grupo el que tendría mayores posibilidades de tener una infección grave por SARS-CoV-2.

Estudios previos y recientes han demostrado que durante los primeros cuatro ciclos de la administración de azacitidina se incrementa el riesgo de infecciones pulmonares (5.1\% de episodios por ciclo), si bien la gran mayoría son de tipo fúngico y bacteriano (5\% de infección por influenza entre los casos con etiología documentada), este tipo de infección en la situación de la pandemia genera problemas en la práctica clínica, ya que implica que el paciente deba acudir al hospital, eventualmente internarse y en algunos casos atenderse en las áreas restringidas a pacientes COVID, si es que la prueba diagnóstica tarda en reportarse ${ }^{9-11}$.

Se deben sopesar los riesgos de llevar pacientes a un hospital lleno de pacientes con COVID-19, ya que se puede realizar atención médica a domicilio verificando signos vitales, así como realizar y revisar estudios de laboratorio para determinar el tipo de tratamiento en los pacientes con SMD; también se pueden cambiar las terapias por otras que puedan autoadministrarse $^{12}$.

La anemia es un problema predominante para la mayoría de los pacientes con SMD. La decisión de transfundir depende de los síntomas de los pacientes en lugar de un resultado de laboratorio, dada la escasez prevista de productos sanguíneos en este tiempo. Muchos pacientes están llegando al límite de su tolerabilidad y retrasando las transfusiones debido al riesgo que conlleva acudir al hospital ${ }^{12}$.

\section{Recomendaciones internacionales}

La National Comprehensive Cancer Network, la European Society for Blood and Marrow Transplantation, la American Society of Clinical Oncology, The Seattle Cancer Care Alliance, el nstitute for Functional Medicine, la Associação Brasileira de Hematologia, Hemoterapia e Terapia Celular y la Sociedade Brasileira de Transplante de Medula Óssea han publicado recomendaciones para pacientes oncológicos que se pueden resumir de la siguiente manera: 
- Implementar un área de control de infecciones.

- Medidas educativas para el personal.

- Minimizar el número de personas que acuden al área donde se atiende a los pacientes oncológicos.

- Posponer los procedimientos quirúrgicos no urgentes.

- Disminuir los tiempos que permanece el paciente en el área hospitalaria.

- La evaluación e identificación de casos sospechosos (triaje respiratorio) en todos los trabajadores del área de oncología y en los pacientes.

- Mejorar el diagnóstico de infecciones virales, incluso si el nivel de sospecha es bajo.

De forma particular en los pacientes con SMD, la Asociación Americana de Hematología recomienda en cuanto al tratamiento de pacientes con SMD, dividirlos de acuerdo con la escala IPSS-R (Revised International Prognostic Scoring System) en:

- SMD de riesgo alto ( $\geq 3.5$ puntos). Iniciar la terapia con hipometilantes sin retraso y sin ajuste de dosis. Si la terapia es adecuadamente tolerada y el paciente tiene respuesta clínica se debe continuar hasta la recaída. También se puede optar por prolongar los intervalos entre ciclos, especialmente si van más de seis meses del tratamiento, al menos a corto plazo ${ }^{12}$.

Para los pacientes que fallan al uso de agentes hipometilantes, los ensayos clínicos son la única opción de tratamiento, en este caso se debe evaluar cada caso de forma individualizada.

El TCPH es la única opción curativa en SMD de riesgo alto. Desafortunadamente, pueden presentarse diversas complicaciones inmediatas y a largo plazo, que incluyen: la enfermedad de injerto contra huésped, rechazo del injerto, toxicidad relacionada con el acondicionamiento, recaída e infecciones. Por lo tanto, durante la pandemia de COVID-19, la decisión de proceder con TCPH en pacientes con SMD pesa sobre múltiples factores; a) estado de la enfermedad del paciente, b) estado funcional, c) carga de casos locales de COVID-19, d) disponibilidad de terapia puente alternativa, e) experiencia del centro de trasplante y f) pautas y regulaciones locales ${ }^{8}$. Si posterior a evaluar riesgo y beneficio se ofrece TCPH, la recomendación es realizar una prueba de reacción en cadena de la polimerasa para SARS-CoV-2 antes del procedimiento tanto al paciente como al donante y dependiendo del resultado retrasar el tratamiento por 2 a 3 semanas $^{8}$.
- SMD riesgo bajo (<3.5). En este grupo el objetivo es disminuir el número de transfusiones, así como los síntomas, y mejorar la calidad de vida, con el uso de los agentes estimulantes de la eritropoyesis (ESA) o luspatercept ${ }^{12}$.

El uso de eltrombopag se puede considerar en pacientes con trombocitopenia grave que han fallado a otras líneas de tratamiento, para disminuir el uso de transfusiones y los eventos de sangrado ${ }^{12}$.

Los pacientes con deleción $5 q$ deben continuar con lenalidomida oral ${ }^{12}$.

\section{Transfusiones}

Diferir las transfusiones basados en la tolerancia y en los factores de riesgo de cada paciente, modificando los umbrales para indicar la transfusión, siendo para el caso de la hemoglobina valores iguales o menores a $7 \mathrm{~g} / \mathrm{dl}$ y para las plaquetas de $10 \mathrm{mil} / \mu \mathrm{l}$ o mayores si hay síntomas de sangrado ${ }^{12}$.

\section{Consultas}

Individualizar de acuerdo con cada caso procurando la telemedicina.

\section{Manejo de neutropenia grave y fiebre}

No debe retrasarse por el alto riesgo de infecciones que ponen en riesgo la vida.

Se recomienda un mayor uso de factor estimulante de colonias de granulocitos y profilaxis antibiótica para reducir el ingreso por neutropenia febril ${ }^{12}$.

\section{Vacunación}

Se sabe que en el contexto de SMD la vacunación produce una respuesta inmunitaria suficiente. La OMS en sus lineamientos relativos a la COVID-19 recomienda continuar la vacunación contra Streptococcus pneumoniae y el virus de la influenza de manera habitual ${ }^{9}$.

\section{Recomendaciones nacionales}

El tratamiento de los pacientes con SMD en la situación de la pandemia por COVID-19 debe tener como premisas:

- Evitar la exposición del paciente al riesgo de acudir al hospital: difiriendo consultas y 
transfusiones, facilitando el surtimiento de recetas en los casos de hospitales públicos, realizando telemedicina (teléfono, correo electrónico, videoconferencia).

- Incrementar las medidas de protección del paciente: confirmando durante la consulta médica los cuidados del paciente y del cuidador.

- Indicar los tratamientos adecuados según corresponda: excepto por el trasplante de progenitores hematopoyéticos, el cual debe realizarse solo cuando se tenga la disponibilidad de la prueba de reacción en cadena de la polimerasa para el SARS-CoV-2 y los donantes de sangre suficientes para cubrir un periodo de hasta cuatro semanas con citopenias graves.

Las recomendaciones para el tratamiento son:

- En los pacientes de riesgo bajo por IPSS-R $(<3.5)$ que estén en vigilancia o en respuesta con el uso de factores estimulantes como ESA, filgrastim 0 análogos trombopoyéticos, se debe continuar el tratamiento de manera habitual. Si son tratados con ciclosporina, debemos indicar la dosis efectiva y procurar la suspensión temprana en caso de falla terapéutica. Hay que evitar la administración de globulina antitimocito, por el efecto que tiene sobre el sistema inmunitario adaptativo.

- En los pacientes de riesgo alto por IPSS-R $(\geq 3.5)$ se debe continuar el tratamiento establecido y ajustar de acuerdo con la respuesta hematológica 0 a las indicaciones del ensayo clínico en el que se encuentren. En los pacientes que inician azacitidina, administrar vía subcutánea, en la dosis habitual, administrando profilaxis en los primeros cuatro ciclos de tratamiento (ciprofloxacino o levofloxacino, posaconazol o voriconazol).

- La vacunación contra el neumococo y el virus de la influenza se encuentra recomendada.

- La terapia transfusional ha sido muy limitada por la pobre disponibilidad de donantes, por lo que debemos tratar de manera puntual con el paciente y familiares la gestión de donantes confiables, sobre todo de aféresis plaquetarias o en el caso de grupos sanguíneos poco frecuentes. El no tener los medios sociales o mediáticos para el aporte de donantes para el paciente específico debe restringir el tratamiento planeado.

- EI TCPH en pacientes candidatos (riesgo pronóstico alto y muy alto) debe planearse considerando el conjunto de paciente-donante-cuidador, tanto por el riesgo de infección pretrasplante como en el postrasplante (primeros seis meses). Además de tener el acceso a pruebas diagnósticas confiables (reacción en cadena de la polimerasa, tomografía computarizada de tórax de alta resolución), la disponibilidad de cuidados intensivos en el caso de infección aguda y los donantes de sangre suficientes. Emplear terapias puente, como hipometilantes o quimioterapia aportarán tiempo adicional para resolver las limitantes logísticas. Finalmente, dado que el riesgo de la COVID-19 en el contexto del trasplante no ha sido definido, contra el riesgo fatal en caso de progresión del SMD, la toma de decisiones requerirá de una participación del paciente y familiares.

\section{Conclusiones}

No existe evidencia concluyente que establezca que los pacientes con SMD tienen mayor riesgo de adquirir infección por el SARS-CoV-2 o que la COVID-19 tenga mayor tasa de letalidad. Sin embargo, conocemos el desenlace del SMD si no llevamos a cabo un tratamiento oportuno; por lo anterior debemos de continuar indicando los tratamientos de manera convencional, incrementando las medidas preventivas de infección tanto del paciente como del cuidador, así como concienciándolos en la importancia de la donación de productos sanguíneos. La realización de un TCPH en la situación actual es muy limitada, por lo que debe optimizarse tomando decisiones con la participación del paciente.

\section{Conflicto de intereses}

Los autores declaran no tener conflictos de interés alguno con el tema de la publicación.

\section{Financiamiento}

No se recibió financiamiento ni patrocinio por ninguna organización o institución.

\section{Responsabilidades éticas}

Protección de personas y animales. Los autores declaran que para esta investigación no se han realizado experimentos en seres humanos ni en animales. 
Confidencialidad de los datos. Los autores declaran que han seguido los protocolos de su centro de trabajo sobre la publicación de datos de pacientes.

Derecho a la privacidad y consentimiento informado. Los autores declaran que en este artículo no aparecen datos de pacientes.

\section{Bibliografía}

1. World Health Organization. Coronavirus disease 2019 (COVID-19). Situation Report - 51 [Internet]. World Health Organization [citado: 31/03/2020]. Disponible en: https://apps.who.int/iris/handle/10665/331475

2. COVID-19 Coronavirus pandemic [Internet]. Worldmeter [citado: 11/04/2020]. Disponible en: https://www.worldometers.info/coronavirus/

3. Jiang $F$, Deng L, Zhang $L$. Review of the clinical characteristics of coronavirus disease 2019 (COVID-19). J Gen Intern Med. 2020;35(5):1545-9.

4. Huang C, Wang Y, Li X, Ren L, Zhao J, Hu Y, et al. Clinical features of patients infected with 2019 novel coronavirus in Wuhan, China. Lancet. 2020;395(10223):497-506
5. Liang W, Guan W, Chen R, Wang W, Li J, Xu K, et al. Cancer patients in SARS-CoV-2 infection: a nationwide analysis in China. Lancet Oncol. 2020;21(3):335-7.

6. Gosian R, Abdou Y, Singh A. COVID-19 and cancer: a comprehensive review. Curr Oncol Rep. 2020;22(5):1-15.

7. Garnica M, Maiolino A. COVID and hematology: special considerations regarding patient safety, gold standard therapies and safety for health care professionals. Hematol Transfus Cell Ther. 2020;42(2):111-2.

8. Sahu KK, Cerny J. Managing patients with hematological malignancies during COVID-19 pandemic. Expert Rev Hematol. 2020;13(8):787-93.

9. Trubiano JA, Dickinson M, Thursky KA, Spelman T, Seymour JF, Slavin MA, et al. Incidence, etiology and timing of infections following azacitidine therapy for myelodysplastic syndromes. Leuk Lymphoma. 2017;58(10):2379-86.

10. Latagliata R, Niscola P, Fianchi L, Aloe Spiriti MA, Maurillo L, Carmosino I, et al. Pulmonary infections in patients with myelodysplastic syndromes receiving frontline azacytidine treatment. Hematol Oncol. 2020;38(2):189-96.

11. Percival ME, Lynch RC, Halpern AB, Shadman M, Cassaday RD, Ujjani $C$, et al. Considerations for managing patients with hematologic malignancy during the COVID-19 pandemic: The Seattle strategy. JCO Oncol Pract. 2020;16(9):571-8.

12. Raza A, Assal A, Ali AM, Jurcic JG. Rewriting the rules for care of MDS and AML patients in the time of COVID-19. Leuk Res Rep. 2020;13:100201. 